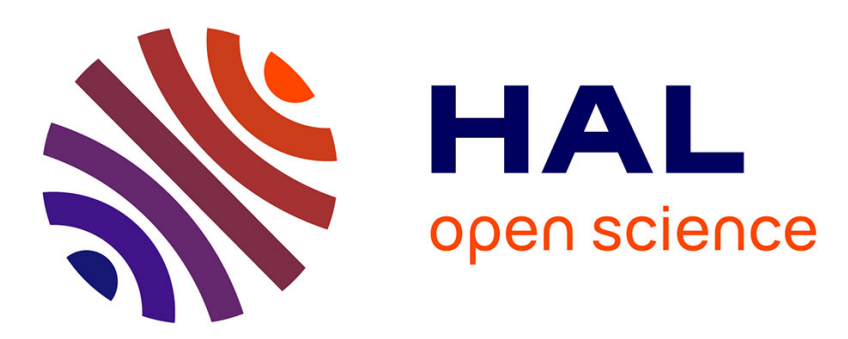

\title{
LMI \& BMI technics for the design of a PI control for irrigation channels
}

Mickael Rodrigues, Yongxin Wu, Samir Aberkane, Valérie dos Santos

\section{To cite this version:}

Mickael Rodrigues, Yongxin Wu, Samir Aberkane, Valérie dos Santos. LMI \& BMI technics for the design of a PI control for irrigation channels. 12th European Control Conference, ECC'13, Jul 2013, Zürich, Switzerland. pp.CDROM. hal-00823551

\section{HAL Id: hal-00823551 \\ https://hal.science/hal-00823551}

Submitted on 17 May 2013

HAL is a multi-disciplinary open access archive for the deposit and dissemination of scientific research documents, whether they are published or not. The documents may come from teaching and research institutions in France or abroad, or from public or private research centers.
L'archive ouverte pluridisciplinaire HAL, est destinée au dépôt et à la diffusion de documents scientifiques de niveau recherche, publiés ou non, émanant des établissements d'enseignement et de recherche français ou étrangers, des laboratoires publics ou privés. 


\title{
LMI \& BMI Technics for the Design of a PI Control for Irrigation Channels
}

\author{
Mickael Rodrigues, Yongxin Wu, Samir Aberkane $†$ Valérie Dos Santos Martins
}

\begin{abstract}
This paper considers the problem of control design for a nonlinear distributed parameter system in infinite dimension which is described by hyperbolic Partial Differential Equations (PDEs) of de Saint-Venant. For describing the dynamic of this nonlinear system over a wide operating range, the Multi-Models approach, which takes into account Linear Time Invariant (LTI) models defined around a set of operating points, has been used. By means of an Internal Model Boundary Control (IMBC), a new design of Proportional Integral (PI) feedback is performed through Bilinear Matrix Inequality (BMI) and Linear Matrix Inequality (LMI) technics. The new results have been simulated and also compared to the previous results, illustrating the new theoretical contribution.
\end{abstract}

\section{Introduction}

Regulation of irrigation channels has received an increasing interest over the last two or three decades. Indeed, water losses in open channels are very large due to inefficient management and control. In order to deliver water, it is important to ensure that water level and the flow rate in the open channel remain at certain values [15]. The difficulty of this regulation problem is that only the gate positions are able to meet performance specifications. So this needs to design boundary control laws satisfying the control objectives. The channel regulation problem has been previously considered in the literature using a wide variety of technics [14]. Studying directly the nonlinear dynamics is also possible as in $[6,13]$. Recent approaches consider the distributed feature of the system. Using the Riemann coordinates approach on the de Saint-Venant equations, stability results are given for a system of two conservation laws, and for system of larger dimension. Lyapunov technics have been used in $[4,6]$. In practice, the physical

${ }^{*}$ M. Rodrigues, M. Wu, and Ms. Dos Santos Martins are with LAGEP, Université de Lyon, Lyon, F-69003, France; Université Lyon 1, CNRS, UMR 5007, LAGEP, Villeurbanne, F-69622, France. Email: surname@lagep.univ-lyon1.fr.

${ }^{\dagger}$ M. Aberkane is with CRAN UMR 7039, Nancy-Université, CNRS, Faculté des Sciences et Technologies-B.P. 70239, F54506 Vandoeuvre-les-Nancy. E-mail: samir.aberkane@cran.uhp-nancy.fr systems are generally represented by nonlinear models. The Multi-Models structure is well adapted for nonlinear systems [1] and for fault diagnosis [2, 17] in finite dimension. Indeed this structure allows to determine a set of linear models defined around some predefined operating points. Each local model is defined as a LTI dynamic system defined for a specific operating point. In the Multi-Models concept, some authors speak about gain scheduling strategy for example in [11] or for interpolated controllers or switching controllers [16].

The use of Multi-Models representation for stability study of system described by nonlinear PDE has been studied in [9]. In this paper, an analysis of the stability of the problem of de Saint-Venant equations is proposed with the use of Multi-Models and Internal Model Boundary Control (IMBC) structure. The stability in Multi-Models framework is often performed by Linear Matrix Inequality (LMI) due to the effectiveness for calculating a unique gain solution for multiple models $[8,9]$. But in control synthesis, BMI provides more general frameworks than LMI.

The paper is organized as follows: firstly, the equations of de Saint-Venant are presented as well as the control problem. Secondly, the linearized systems are developed around equilibrium sets which depend on the space variable. Third part of the paper is dedicated to the design of the feedback gain by LMI\&BMI technics which ensures the stability of the closed loop system in finite dimension. The last section is dedicated to the simulations and comparison with previous works.

\section{Problem statement about channel regu- lation}

Let us consider the following class of water channels represented on figure (1), i.e. a reach of an open channel delimited by underflow gates where $Q(x, t)$ and $Z(x, t)$ are the state variables and stand for the water flow rate and the height of water in the channel respectively, $L$ is the length of the reach from the upstream $x=0$ to the downstream $x=L, U_{u p}=U_{0}(t), U_{d o}=U_{L}(t)$ are the opening of the gates at upstream and downstream respectively. The regulation problem concerns the stabilization of the water flow rate and/or the height of the water around an equilibrium for a reach denoted by $\left(Z_{e}(x), Q_{e}(x)\right)$. A linear model with variable coeffi- 
cients can be deduced from the non-linear PDE, in order to describe the variation of the water level and flow for an open channel. Let us recall these models.

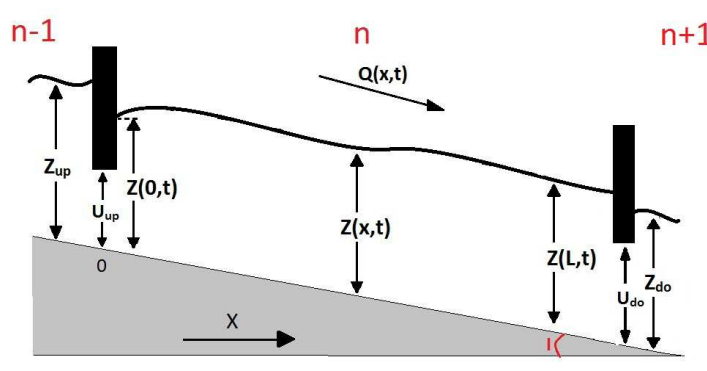

Figure 1. Channel scheme: 2 underflow gates

\subsection{A model of a reach}

The channel is supposed to be rectangular and to have a sufficient length $L$ such that hydraulic conditions are assumed following the classical 1D assumptions. Nonlinear PDE of de Saint-Venant which describe the flow on the channel are the following $[8]^{1}$ :

$$
\begin{aligned}
& \partial_{t} Z=-\partial_{x} \frac{Q}{b} \\
& \partial_{t} Q=-\partial_{x}\left(\frac{Q^{2}}{b Z}+\frac{1}{2} g b Z^{2}\right)+g b Z(I-J), \\
& Z_{0}(x)=Z(x, 0), Q_{0}(x)=Q(x, 0)
\end{aligned}
$$

$\forall x \in \Omega=\left(x_{u p}, x_{d o}\right)=(0, L), t>0$, where $I$ is the slope, $b$ is the channel width, $g$ is the gravity constant. $J$ is the friction slope from the formula of Manning-Strickler. (3) are the initial conditions. The limit conditions considered here are the multivariable case, $\forall x \in \Gamma=\partial \Omega$ the boundary of $\Omega$ on figure (1):

$$
Q(x, t)=U(t) \Psi_{i}(Z(x, t))
$$

with $\Psi_{i}(Z)=\kappa_{i} \sqrt{2 g\left(Z_{u p}-Z_{d o}\right)} . Z_{u p} Z_{d o}$ are the water height at the upstream of the considered gate, resp. at downstream of the considered gate, $\kappa_{i}$ is the product of the channel width with the water flow rate coefficient of the gate $n^{0} i, U(t)$ is the control of the considered gate. The variable to control is the height of water at downstream, i.e. $Z(L)$, considering two underflow gates.

\subsection{A regulation model}

An equilibrium state $\left(\partial_{t}(.) \equiv 0\right)$ of the system satisfies the following equations:

$$
\partial_{x} Q_{e}=0, \partial_{x} Z_{e}=g b Z_{e} \frac{I+2 J_{e}+\frac{4}{3} J_{e} \frac{1}{1+2 Z_{e} / b}}{g b Z_{e}-Q_{e}^{2} / b Z_{e}^{2}}
$$

The fluvial case is considered and it follows that:

$$
Z_{e}>\sqrt[3]{Q_{e}^{2} /\left(g b^{2}\right)}
$$

\footnotetext{
${ }^{1} \partial_{\theta} f=\partial f / \partial \theta$
}

Let us denote that $Q_{e}$ is constant but $Z_{e}$ depends on variable space. With $\xi(t)=(z(t), q(t))^{t}$, the linearized model around an equilibrium point $\left(Z_{e}(x), Q_{e}(x)\right)^{t}$ is:

$$
\begin{gathered}
\partial_{t} \xi(x, t)=\left[A_{1}(x) \partial_{x}+A_{2}(x)\right] \xi(x, t)=\mathscr{A} \xi(x, t) \\
\xi(0, t)=\xi_{0}(t) \\
q\left(x_{u p}\right)=U_{u p, e} \partial_{z} \Psi_{1}\left(Z_{e}\left(x_{u p}\right)\right) z\left(x_{u p}\right)+u_{u p} \Psi_{1}\left(Z_{e}\left(x_{u p}\right)\right) \\
q\left(x_{d o}\right)=U_{d o, e} \partial_{z} \Psi_{2}\left(Z_{e}\left(x_{d o}\right)\right) z\left(x_{d o}\right)+u_{d o} \Psi_{2}\left(Z_{e}\left(x_{d o}\right)\right)
\end{gathered}
$$

where $U_{u p, e}, U_{d o, e}$ are the gates opening for the upstream and downstream at the equilibrium and $u_{u p}(t)$, $u_{d o}(t)$ are the variation of these gates opening to be controlled. The matrices $A_{1}(x), A_{2}(x)$ are given by:

$A_{1}(x)=\left(\begin{array}{cc}0 & -1 / b \\ -a_{2} & -a_{3}\end{array}\right), A_{2}(x)=\left(\begin{array}{cc}0 & 0 \\ a_{4} & -a_{5}\end{array}\right)$

with $a_{2}(x)=g b Z_{e}-\frac{Q_{e}^{2}}{b Z_{e}^{2}(x)}, a_{5}(x)=\frac{2 g b J_{e}(x) Z_{e}(x)}{Q_{e}}$,

$a_{4}(x)=g b\left(I+2 J_{e}(x)+\frac{\frac{4}{3} J_{e}(x)}{1+2 Z_{e}(x) / b}\right), \quad a_{3}(x)=\frac{2 Q_{e}}{b Z_{e}(x)}$.

The control problem is to find the variations of $u_{u p}(t)$ and $u_{d o}(t)$ at extremities $x=x_{u p}$ and $x=x_{d o}$ resp. of the reach such that downstream water level, $Z\left(x_{d o}, t\right)=Z(L, t)$ (measured variable), tracks a reference signal $r(t) . r(t)$ is chosen for all cases either constant or non-persistent (a stable step answer of a nonoscilatory system). In this paper, the Internal Model Boundary Control (IMBC) [7] is adopted (figure (2)). This control strategy integrates the process model in real time and allows to regulate the water height in all the points of the channel by taking into account the error between the linearized model and the real system (or the NL model for the simulations).

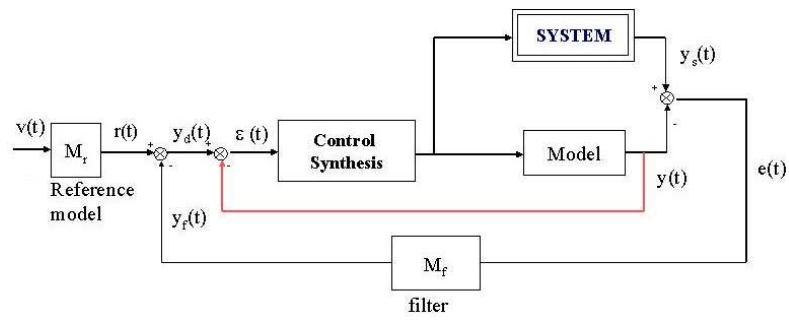

Figure 2. IMBC structure

\subsection{Stability of the system}

The equation (7) describes the dynamic of the system in open loop. In this representation, the state vector $\xi(x, t)$ is not explicitly linked with the boundary control. In order to design an output feedback, an operator $D$ of distribution of the boundary control is introduced, it is a bounded operator such that $\operatorname{Im}(D)=\operatorname{Ker}(\mathscr{A})$ and $D u \in$ $D(\mathscr{A}),[7]$ :

$$
\xi(x, t)=\varphi(x, t)+D u(t)
$$


This operator is naturally null in the domain of $\mathscr{A}(x)$ as it is active only on the boundary of the domain. This change of variables allows to get a Kalman representation for the system:

$$
\begin{array}{r}
\partial_{t} \varphi(x, t)=\mathscr{A}(x) \varphi(x, t)-D \dot{u} \\
\varphi(x, 0)=\varphi_{0}(x)=\xi_{0}(x)-D u(0)
\end{array}
$$

It has been proved that the open loop system described above is exponentially stable [7], as the operator of the linearized system in infinite dimension generates an exponentially stable $C_{0}$-semigroup. All the more, under a control $u \in C^{\alpha}([0, \infty], U)^{2}$, conditions on the tuning parameters are also given to ensure the stability of the closed loop system. Those theoretical results have been coupled with simulations and experimentations that have confirmed this approach [7]. Those experimentations have risen up the limitations due to the linearization around an equilibrium state and a first attempt of a Multi-Models experimentations have been realized with success (figure (5)) but it was not optimal and no theoretical proof has been given. A first approach for an integral control then for a PI control in [8] have been done with gains of the proportional and of the integrator equals. The aim of this paper is to develop the previous results with the proportional gain different from the integrator one. In order to control the water level over a wide operating range, sufficient conditions are presented to preserve the stability of this system all along the operating set, under a PI control, in finite dimension.

\subsection{A Multi-Models representation of Saint- Venant's Equation}

The Multi-Models structure [17] allows to control the system over a wide operating range because it takes into account the different sub-models which can be activated under different operating levels. The representation of de Saint-Venant's PDE around $N$ operating points by the Multi-Models approach is defined by the following equations:

$$
\begin{gathered}
\partial_{t} \xi(x, t)=\sum_{i=1}^{N} \mu_{i}(\zeta(t)) \mathscr{A}_{i}(x) \xi(x, t) \\
\mathscr{A}_{i}(x)=A_{1, i}(x) \partial_{x}+A_{2, i}(x) \\
\xi_{0}(x)=\xi(x, 0)
\end{gathered}
$$

where $\mathscr{A}_{i}(x)$ is the operator which corresponds to the $i^{t h}$ equilibrium state. $\zeta(t)$ is a function depending of some decision variables directly linked with the mesurables states variables and eventually to the input. $\mu_{i}(\zeta(t)) \in$ $(0,1)$ is the weighting functions which determines the sub-model for the control law synthesis depending of

\footnotetext{
${ }^{2}$ Regularity coefficient is generally taken as $\alpha=2$
}

the output height of the process $z_{L}$. In the following section, the synthesis of a control law by LMI \& BMI technics is considered: a proportional integral control and the synthesis of the gains which preserve the stability of the system all over the operating range are deduced from BMI technics.

\section{Stability study}

\subsection{Closed-loop structure for a proportional integral feedback}

For a control with an output feedback, $u(t)=$ $K_{\text {int }} \int \varepsilon(\tau) d \tau+K_{p r} \varepsilon(t)$, where $K_{\text {int }}$ and $K_{p r}$ are defined as the gains, it follows that [7]:

$$
\begin{gathered}
\varepsilon(t)=r(t)-y(t) \\
u(t)=K_{\text {int }} \int[r(\tau)-y(\tau)] d \tau+K_{p r}[r(t)-y(t)]
\end{gathered}
$$

with $y(t)=C(\xi(x, t)+E q(x, t))$ where $E q(x, t)=$ $\sum_{i=1}^{N} \mu_{i}(\zeta(t))\left(z_{e, i}(x, t) q_{e, i}\right)^{t}$ is the equilibrium state, and for example $C E q(x, t)=\sum_{i=1}^{N} \mu_{i}(\zeta(t)) z_{e, i}(L)$ if the aim is to regulate the water level at $x=L$. $C$ is the the output operator is the Kalman structure.

$$
y(t)=C \varphi(x, t)+C E q(x, t)+C D u(t)
$$

and by replacing $y(t)$ into the control equation (14):

$$
\begin{aligned}
& u(t)=K_{p r}[r(t)-(C \varphi(x, t)+C E q(x, t)+C D u(t))] \\
& +K_{\text {int }} \int[r(\tau)-(C \varphi(x, \tau)+C E q(x, \tau)+C D u(\tau))] d \tau \\
& \Rightarrow \dot{u}(t)=K_{\text {int }}[r(t)-C \varphi(x, t)-C E q(x, t)-C D u(t)] \\
& +K_{p r}\left[\dot{r}(t)-C \dot{E} q(x, t)-C \mathscr{A}_{i}(x) \varphi\right]
\end{aligned}
$$

In each local model, $E q(x, t)$ is a piecewise function $(\dot{E} q(x, t)=0)$, it is also the case of $r(t)$. So $\dot{u}$ can be simplified into the following form:

$$
\begin{aligned}
\dot{u}(t)= & K_{i n t}[r(t)-C \varphi(x, t)-C E q(x, t)-C D u(t)] \\
& -K_{p r} C \mathscr{A}_{i}(x) \varphi
\end{aligned}
$$

and using $\dot{u}$ into the equation (9), the expression of closed-loop is then:

$$
\begin{gathered}
\partial_{t} \varphi(x, t)=\sum_{i=1}^{N} \mu_{i}(\zeta(t))\left[\left(A_{i}+D K_{i n t} C+D K_{p r} C A_{i}\right) \varphi(x, t)\right. \\
\left.+D K_{\text {int }}(C D u(t)+C E q(x, t)-r(t))\right]
\end{gathered}
$$

Let define:

$$
\tilde{K}_{\text {int }}=D K_{\text {int }}, \tilde{K}_{p r}=D K_{p r}
$$

The equation (17) can be written as:

$$
\begin{aligned}
\partial_{t} \varphi(x, t)= & \sum_{i=1}^{N} \mu_{i}(\zeta(t))\left[\left(\mathscr{A}_{i}+\tilde{K}_{i n t} C+\tilde{K}_{p r} C \mathscr{A}_{i}\right) \varphi(x, t)\right. \\
& \left.+\tilde{K}_{\text {int }}(C D u(t)+C E q(x, t)-r(t))\right] \\
& =\sum_{i=1}^{N} \mu_{i}(\zeta(t)) \mathscr{M}_{i}(x, t)
\end{aligned}
$$


The conditions which provide the stability are ensured by using a quadratic Lyapunov function in order to guarantee the convergence of the water height to the reference $r(t)$, over the widest operating range.

\subsection{Stability study with a quadratic Lyapunov function}

Let us consider:

$$
V(\varphi(x, t), t)=<\varphi(x, t), P \varphi(x, t)>
$$

where $<., .>$ is the considered inner product. The Multi-Models representation of the linearized PDE of de Saint-Venant defined by equation (17) is asymptotically stable if there exists a $P>0$, such that ${ }^{3}$ : For finite dimensional systems $(P>0$ is a matrix $)$ and infinite dimensional systems ( $P>0$ is an operator) [5] resp.:

$$
\begin{array}{r}
\dot{V}(\varphi(x, t), t)<0 \Leftrightarrow<\dot{\varphi}, P \varphi>+<\varphi, P \dot{\varphi}><0 \\
<\dot{\varphi}, P \varphi>+<\varphi, P \dot{\varphi}>=-<\varphi, \varphi>
\end{array}
$$

The LMI technics are usually defined for finite dimensional systems, the authors begin to give some proof of the LMI technics application for the stability study to infinite dimensional system [9, 8]. The authors of [19] have used LMI technics for the stability study to a non-linear distributed parameter system with a development of fuzzy state-feedback control. The authors in [10] have worked on the stability of switching systems in infinite dimension and they are still working to link those mathematical results to the LMI approach. The main difference here between the stability result in finite and infinite dimension, is located in the inequality of the Lyapunov function for finite dimensional systems (21) and equality for infinite ones (22). This equality complexity can be removed in some cases: as for example for operators with compact resolvent $[5,7]$ and in this case the same inequality from finite dimension is a sufficient and necessary condition for the infinite dimensional case: it is a question of satisfying the spectral growth assumption [9]. Moreover for the equations of de Saint-Venant, it has been shown that the operator has a compact resolvent [7] so it satisfies the spectral growth assumption. Then, taking account of (17)-(22), it follows that one has to prove the inequality:

$$
<\mathscr{M}_{i}, P \varphi>+<\varphi, P \mathscr{M}_{i}><0, \quad \forall i
$$

where $\mathscr{M}_{i}$ is defined in (19). The development of this inequality leads us to consider an inequality for each local system of index i such that:

$$
<\left[\mathscr{A}_{i}+\tilde{K}_{i n t} C+\tilde{K}_{p r} C \mathscr{A}_{i}\right] \varphi(x, t), P \varphi(x, t)>
$$

\footnotetext{
${ }^{3}$ We suppose that $\partial_{t} \phi=\dot{\phi}$ whatever the function $\phi$.
}

$$
\begin{aligned}
& +<\tilde{K}_{\text {int }}[C D u(t)+C E q(x, t)-r(t)], P \varphi(x, t)> \\
& +<\varphi(x, t), P\left[\mathscr{A}_{i}+\tilde{K}_{\text {int }} C+\tilde{K}_{p r} C \mathscr{A}_{i}\right] \varphi(x, t)> \\
& +<\varphi(x, t), P \tilde{K}_{\text {int }}[C D u(t)+C E q(x, t)-r(t)]><0
\end{aligned}
$$

In the inequality (24), which defines the stability condition of the system $\forall i$, the control parameter $u$ appears in this inequality and it is a difficulty for the design of the gains $\tilde{K}_{i n t}, \tilde{K}_{p r}$. A first approach was made in [8] with $K_{p r}=K_{i n t}$. Here, in this paper $K_{i n t}$ is considered different of $K_{p r}$. It has been proved that a good choice of $K_{\text {int }}$ in [7] based on semi-group theory is $K_{\text {int }}=-\alpha_{i}[C D]^{\dagger}(\dagger$ represent the right pseudo-inverse) and $K_{p r}=\alpha_{p}[C D]^{\dagger}$. $\alpha_{i}$ and $\alpha_{p}$ are defined in [7]. So one can suppose that $\exists \beta \in \mathbb{R}$ such that $K_{p r}=\beta K_{\text {int }}$, i.e. $\tilde{K}_{p r}=\beta \tilde{K}_{\text {int }}$ (cf. (18)). Then the equation (24) becomes:

$$
\begin{aligned}
& <\left[\mathscr{A}_{i}+\tilde{K}_{i n t} C+\beta \tilde{K}_{i n t} C \mathscr{A}_{i}\right] \varphi(x, t), P \varphi(x, t)> \\
& +<\tilde{K}_{i n t}[C D u(t)+C E q(x, t)-r(t)], P \varphi(x, t)> \\
& +<\varphi(x, t), P\left[\mathscr{A}_{i}+\tilde{K}_{i n t} C+\beta \tilde{K}_{i n t} C \mathscr{A} i\right] \varphi(x, t)> \\
& +<\varphi(x, t), P \tilde{K}_{i n t}[C D u(t)+C E q(x, t)-r(t)]><0
\end{aligned}
$$

The term $[C D u(t)+C E q(x, t)-r(t)]$ of (25) has been treated in [9] and it has been proved that:

$$
\varphi^{T} P \tilde{K}_{\text {int }}(C D u+C E q-r) \leq \alpha \varphi^{T} P \tilde{K}_{\text {int }} C \varphi
$$

with $\alpha=(k+1) \varepsilon_{\left(\varphi^{T} P \tilde{K}_{\text {int }} C \varphi\right)}$ and $\varepsilon_{f(x)}=\operatorname{sign}(f(x))$. (25) is then implied by (27)

$$
\begin{aligned}
& \varphi(x, t)^{T}\left[\mathscr{A}_{i}^{T} P+P \mathscr{A}_{i}+\gamma W_{i n t} C+\gamma C^{T} W_{i n t}^{T}\right. \\
& \left.+\beta W_{i n t} C \mathscr{A}_{i}+\beta\left(C \mathscr{A}_{i}\right)^{T} W_{i n t}^{T}\right] \varphi(x, t)<0
\end{aligned}
$$

with $\gamma=1+\alpha, W_{i n t}=P \tilde{K}_{i n t}, \tilde{K}_{p r}=\beta \tilde{K}_{i n t}$. The problem is that (27) is a BMI in infinite dimension, for which the tools do not exist as long as we know. In the following proposition, the authors use the methods in finite dimension to ensure the stability of the closed-loop system (19).

Proposition 1 If there exists a symmetric positivedefinite matrix $P$, matrices $W_{\text {int }}$ and $W_{p r}$, the scalars $\sigma, \gamma \in \mathbb{R}$, such that:

$$
\begin{gathered}
{\left[\begin{array}{cc}
\mathscr{A}_{i}^{T} P+P \mathscr{A}_{i}+\gamma W_{i n t} C+\gamma C^{T} W_{i n t}^{T} & W_{i n t} \\
W_{i n t}^{T} & \sigma^{-1}
\end{array}\right]<0} \\
{\left[\begin{array}{cr}
\mathscr{A}_{i}^{T} P+P \mathscr{A}_{i}+\gamma W_{i n t} C+\gamma C^{T} W_{i n t}^{T} & \left(C \mathscr{A}_{i}\right)^{T} \\
C \mathscr{A}_{i} & \sigma^{-1}
\end{array}\right]<0}
\end{gathered}
$$

then, the closed-loop system (19) under the PI control law (14) is stable.

Proof: The inequality (27) has two variables: $\beta$ and $W_{\text {int }}$ that lead to a BMI (Bilinear Matrix Inequality) problem. As BMI is a non-convex constraint, we can not use the convex optimization methods. So the idea 
is to derive two LMIs which are equivalent to this BMI. The BMI problem can thus be solved as a LMI problem by convex optimization method. By using Schür complement [3], the two inequalities (28-29) are sufficient to prove (30)

$$
G-\sigma U^{T} U<0, G-\sigma V^{T} V<0, \forall \sigma \in \mathbb{R}
$$

with $G=\mathscr{A}_{i}^{T} P+P \mathscr{A}_{i}+\gamma W_{\text {int }} C+\gamma C^{T} W_{i n t}^{T}, U^{T}=W_{\text {int }}$, $X=\beta, V=C \mathscr{A}_{i}$. The Finsler's lemma [18] proves that (30) is equivalent to (31)

$$
U^{\perp T} G U^{\perp}<0, \quad V^{\perp T} G V^{\perp}<0
$$

where $U^{\perp}$ and $V^{\perp}$ are orthogonal complements of $U$ and $V$ respectively, i.e. $U^{\perp} U=0$ and $V^{\perp} V=0$. Then based on the Elimination Lemma [3], the inequality (31) is equivalent to the following inequalities:

$$
G+U^{T} X V+V^{T} X U<0 \Leftrightarrow(25)
$$

The two LMIs (28) and (29) allow to solve the BMI problem (27) which can be solved by convex optimization method and determine parameter $\sigma^{-1}$, matrices $W_{\text {int }}$ and $P>0$. After, and based on the numerical values of $W_{\text {int }}$ and $P$, the only unknown parameter is $\beta$. So, one can determine this parameter $\beta$ by solving inequality (27). Then, the gains $\tilde{K}_{\text {int }}=P^{-1} W_{\text {int }}$ and $\tilde{K}_{p r}=\beta \tilde{K}_{\text {int }}$ can be obtained by solving LMI (28-29).

Now, the gains $\tilde{K}_{\text {int }} \neq \tilde{K}_{p r}$ have been implemented in simulations so as to compare the dynamic of the closedloop system versus previous results. The aim is to compare the simulated curves obtained with this method and the ones obtained on the experimental benchmark [7] and the simulations with the gains $\tilde{K}_{i n t}=\tilde{K}_{p r}[8,9]$.

\section{Simulations results}

Firstly, let describe the benchmark used for the simulations in a rectangular micro-channel of Valence (France), which are described in the following subsections. The simulations are based on a Chang and Cooper scheme, for more details see [6,9].

\subsection{Parameters of the micro-channel}

For the study of the micro-channel of Valence, the following set of parameters is considered where the data are defined such that: $L=6.45 \mathrm{~m}$ is the length of the channel, $b=0.1 \mathrm{~m}$ is the width of the channel, $N=20$ is the number of the discretizated points, the slope $I=1.6 \% Z_{L}$ is the water height to regulate, such that $z_{\min }<Z<0.2 m, \forall Z(x, t)$; where $z_{\min }$ is the minimum critical fluvial water level, $0.2 \mathrm{~m}$ is the channel height. The equilibria profiles have been chosen such that the calculated control law from the local models can be efficient over all the operating range of the water height [7]. Let notice that it has been experimentally verified that a local model is valid around $\pm 20 \%$ of an equilibrium profile. In order to assign references which are included between $z_{\min }=0.06 \mathrm{~m}$ and $0.2 \mathrm{~m}$, the operating points at $x=0$ are the following: In this application, the

Table 1. Initial set points for the simulations

\begin{tabular}{|l|l|l|}
\hline$Z_{e 1}(x=0)$ & $Z_{e 2}(x=0)$ & $Z_{e 3}(x=0)$ \\
\hline $0.06219 m$ & $0.10149 m$ & $0.15819 m$ \\
\hline
\end{tabular}

weighting function $\mu_{i}(\zeta(t))$ is equal to 1 if the output's height is included into the validity domain of the model and 0 in the other case for each operating state. The parameter $\zeta(t)$ exclusively depends on the output which is the only one variable of decision in this precise case.

\subsection{Simulations of the micro-channel}

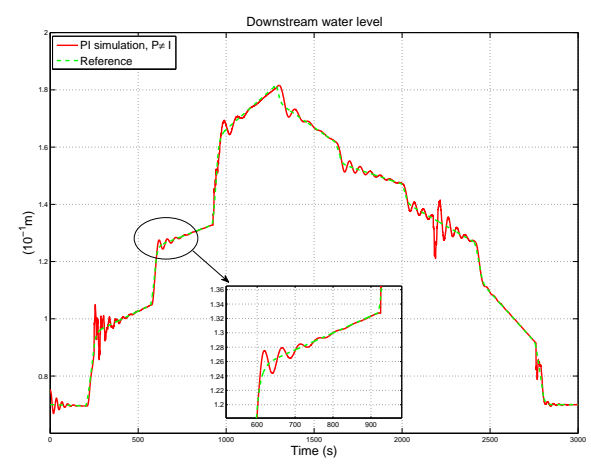

Figure 3. Variations along the valued domain

These results are obtained from an IMB Control and a Multi-Models approach with a LMI gain previously calculated. Figure (3) shows that the output converges to the reference even is this one strongly varies (variations $>100 \%$ towards the initial conditions). The references tracks a dynamic and one can see that the water tracks correctly to reference. The curves that describe the upstream and downstream opening gates of the reach are given by figure (4). The convergence of the output to the reference is ensured even when the reference is decreasing or increasing. The following simulations compare a PI controllers (gains $K_{\text {int }}=K_{p r}$ [8]) and the new PI controllers (gains $K_{\text {int }} \neq K_{p r}$ ), these last gains have been calculated by the LMI approach, the first one with traditional LMI technics, the second by the method of BMI, figure (5). One can observe on figure (5), that the convergence of the new PI controllers is better than the one obtained by PI controllers with $K_{\text {int }}=K_{p r}$ [8], and the overshoot is less too. It is also better than the experimental PI which has been firstly implemented. 


\section{Conclusion}

First attempts of a Multi-Models approach on irrigation channels control, through an IMBC structure, have been realized some years ago [7]. Good experimental results, but without theoretical approach, were obtained and have shown promising results. The first theoretical results in order to tune the feedback gain through LMI have been raised in the case of an integral controller in [9]. A preliminary results of a PI controller in a particular case $\left(K_{\text {int }}=K_{p r}\right)$ have been published [8]. In this paper, the authors take into account the more general case of PI controller with $K_{\text {int }} \neq K_{p r}$, and synthesize a new feedback gain of this PI controller by solving a BMI problem in finite dimension. Simulations have shown a better performance through this new PI feedback controller designed by BMI \& LMI than the previous results. The extension of those works is to extend the tools of BMI technics to the infinite dimension.

\section{References}

[1] Athans M, Fekri S, Pascoal A. 2005. Issues on robust adaptive feedback control. In: Proc. 16th IFAC World Congress, Prague, Czech Republic.

[2] Bhagwat A, Srinivasan R, Krishnaswamy P R. 2003. Multi-linear model-based fault detection during process transitions. Chem. Eng. Science 58, 1649-1670.

[3] Boyd S, El Ghaoui L, Feron E, Balakrishnan V. 1994. Linear Matrix Inequalities in System and Control Theory, Society for Indus. and Appl. Math., Philadelphia, USA.

[4] Coron J M, d'Andréa Novel B, Bastin G. 2007. A strict lyapunov function for boundary control of hyperbolic systems of conservation laws. IEEE Transactions on Automatic Control 52(1), 2-11.

[5] Curtain R F, Zwart H . 1995. An introduction to Infinite Dimensional Linear Systems, Springer Verlag, New York.

[6] Dos Santos V, Prieur C. 2008. Boundary control of open channels with numerical and experimental validations. IEEE TCST 16, 1252-1264.

[7] Dos Santos V, Toure Y, Mendes E, Courtial E. 2005. Multivariable boundary control approach by internal model, applied to irrigations canals regulation. In: Proc. 16th IFAC World Congress, Prague, Czech Republic.

[8] Dos Santos Martins V, Rodrigues M. 2011. A Proportional Integral Feedback for Open Channels Control Trough LMI Design, 18th IFAC World Congress, August 28 - September 2, 2011, Milano, Italy.

[9] Dos Santos Martins V, Rodrigues M, Diagne M. 2012. A Multi-Models Approach of Saint-Venant's Equations: A Stability study LMI. Int. Journal of Applied Mathematics and Computer Science, Vol.22, No.3, September,2012.

[10] Hante F, Sigalotti M. 2010. Existence of common lyapunov functions for infinite-dimensional switched linear systems, 49th IEEE Conference on Decision and Control (CDC), Atlanta, GA pp.5668-5673. ISSN:0743-1546.

[11] Leith D J, Leithead W E. 2000. Survey of gainscheduling analysis and design. International Journal of
Control 73 (11), 1001-1025.

[12] Li T. 1994. Global Classical Solutions for Quasilinear Hyperbolic Systems. Research in Applied Mathematics. Masson and Wiley, Paris, Milan, Barcelona.

[13] Litrico X, Fromion V, Baume J P, Arranja C, Rijo M. 2005. Experimental validation of a methodology to control irrigation canals based on Saint-Venant equations. Control Engineering Practice 13, 1425-1437.

[14] Malaterre P O, Rogers D, Schuurmans J. 1998. Classification of canal control algorithms. J. of Irrigation and Drainage Engineering 124(1), 3-10.

[15] Mareels I, Weyer E, Ooi S, Cantoni M, Li Y, Nair G. 2005. Systems engineering for irrigation systems: Successes and challenges. An. Rev. in Con. 29(2), 191-204.

[16] Narendra K, Balakrishnan J, Kermal M. 1995. Adaptation and learning using multiple models, switching and tuning. IEEE Contr. Syst. Mag., 37-51.

[17] Rodrigues M, Theilliol D, Adam-Medina M, Sauter D. 2008. A fault detection and isolation scheme for industrial systems based on multiple operating models. Control Engineering Practice 16, 225-239.

[18] Skelton R, Iwasak T, Grigoriadis K. 1997. A Unified Algebraic Approach to Linear Control Design. Taylor and Francis, London, UK.

[19] Wang J W, Wu H N, Li H X. 2011. Distributed fuzzy control design of nonlinear hyperbolic PDE systems with application to nonisothermal plug-flow reactor, IEEE T. Fuzzy Systems 19(3):514-526.

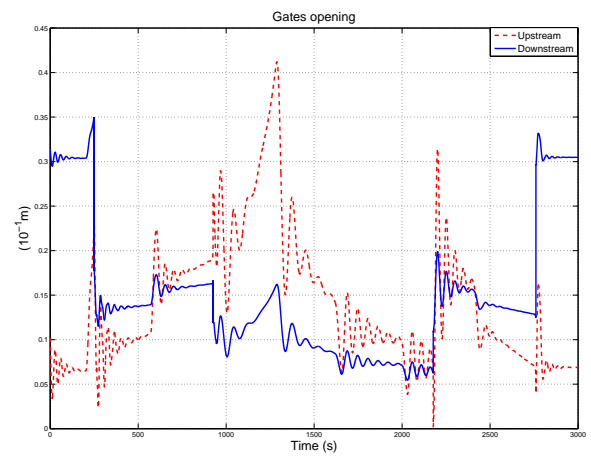

Figure 4. Gates opening

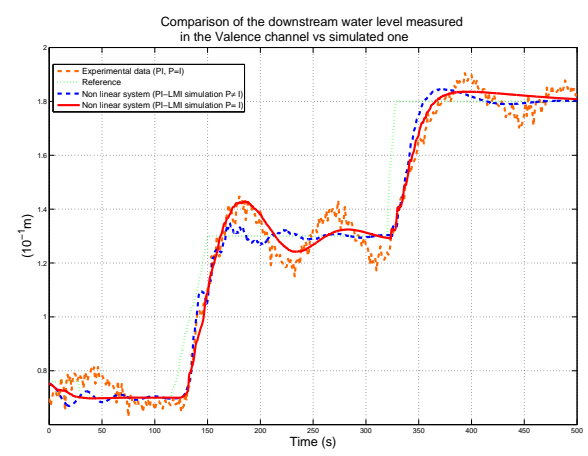

Figure 5. Experiment vs simulation 